\title{
One-Step-Ahead Predictive Control for Hydroturbine Governor
}

\author{
Zhihuai Xiao, ${ }^{1}$ Suili Meng, ${ }^{1} \mathrm{Na} \mathrm{Lu}^{2}{ }^{2}$ and O. P. Malik ${ }^{3}$ \\ ${ }^{1}$ School of Power and Mechanical Engineering, Wuhan University, Wuhan, Hubei 430072, China \\ ${ }^{2}$ School of Water Resources and Architectural Engineering, Northwest A\&F University, Xian, Shaanxi 712100, China \\ ${ }^{3}$ Department of Electrical and Computer Engineering, University of Calgary, Calgary, AB, Canada T2N 1N4
}

Correspondence should be addressed to Zhihuai Xiao; xiaozhihuai@126.com

Received 24 December 2014; Accepted 23 January 2015

Academic Editor: Yun-Bo Zhao

Copyright (C) 2015 Zhihuai Xiao et al. This is an open access article distributed under the Creative Commons Attribution License, which permits unrestricted use, distribution, and reproduction in any medium, provided the original work is properly cited.

\begin{abstract}
The hydroturbine generator regulating system can be considered as one system synthetically integrating water, machine, and electricity. It is a complex and nonlinear system, and its configuration and parameters are time-dependent. A one-step-ahead predictive control based on on-line trained neural networks (NNs) for hydroturbine governor with variation in gate position is described in this paper. The proposed control algorithm consists of a one-step-ahead neuropredictor that tracks the dynamic characteristics of the plant and predicts its output and a neurocontroller to generate the optimal control signal. The weights of two NNs, initially trained off-line, are updated on-line according to the scalar error. The proposed controller can thus track operating conditions in real-time and produce the optimal control signal over the wide operating range. Only the inputs and outputs of the generator are measured and there is no need to determine the other states of the generator. Simulations have been performed with varying operating conditions and different disturbances to compare the performance of the proposed controller with that of a conventional PID controller and validate the feasibility of the proposed approach.
\end{abstract}

\section{Introduction}

Hydroturbine governor (HTG) provides the basic control in hydropower stations to ensure the reliability and the quality of electricity supply. The conventional hydroturbine governor adopted by most utilities is a proportional, integral, and derivative (PID) type controller based on linear control theory. It has simple structure with flexibility and is easy for implementation, and thus it has made a great contribution in enhancing the quality of electrical supply [1].

The hydroturbine generator regulating system can be considered as one system synthetically integrating water, machine, and electricity. It is a complex and nonlinear system, and its configuration and parameters are time-dependent [2]. Nonlinear models are required when speed and power changes are large during an islanding, load rejection, and system restoration conditions. A nonlinear model should include the effect of water compressibility, that is, inclusion of transmission-line-like reflections which occur in the elasticwalled pipe carrying compressible fluid $[3,4]$.
This creates discrepancies between the mathematical linear model of the hydroturbine generator regulating system and the physical nonlinear plant. Therefore, with the conventional linear control theory based PID controller, it is difficult to realize the desired control performance over wide operating conditions of the power plant [5]. To yield satisfactory control performance, it is desirable to develop a controller that considers the nonlinear nature of the plant and has the ability to adjust its parameters on-line according to the environment in which it is working, that is, track the plant operating conditions [6].

To meet this requirement, a large amount of research has been conducted on the hydroturbine generator regulating system. Numerous methods for PID tuning have been reported in the literature [7]. For example, to realize the parameter optimization of PID controller, an orthogonal test strategy is adopted in $[8,9]$ for hydroturbine control application. In this approach, a control performance index that depends on control parameters $\mathrm{KP}, \mathrm{Ki}$, and $\mathrm{Kd}$ is defined. Each of these parameters is considered under various levels as 
a discrete variable. An optimization algorithm is developed to search for better control parameters in the neighbouring space of the present one. However, many of these approaches lack one or more of the three basic and important features that a controller should have, that is, simplicity of structure, fast acting (low computation time), and adaptability.

Neural networks (NNs) have been applied very successfully in the identification and control of dynamic systems. The universal approximation capabilities of the multilayer perceptron make it a popular choice for modeling nonlinear systems and implementing general-purpose nonlinear controllers [10-13]. The concept of intelligent tuning of PID controller is presented in $[14,15]$. The use of adaptive learning control schemes is discussed in [14] and the improved dynamic performance of the intelligent PID controller over the conventional PID is presented in [15]. The developed intelligent PID controller is based on anthropomorphic intelligence. Among various types of NNs used in the hydroturbine generator regulating system, the feed-forward multilayer NN is the most commonly used. This is mainly due to the computational efficiency of the back propagation algorithm [12] and the versatility of the three-layer feed-forward NN in approximating an arbitrary static nonlinear function [16]. However, little work has been reported on the use of NNs for hydroturbine generator regulating system for real-time control.

The theory and algorithm of predictive control have achieved great development in the industrial process control after thirty years' application and study. It has been introduced to the optimal control of hydroturbines by lots of experts. Jones and Mansoor [17], for example, applied predictive feed-forward control to the power target signal tracking in part load condition, and the result shows that predictive feed-forward control can achieve a substantial improvement in tracking a power target when a hydroelectric station operates in part load condition mode. However, the power station they used is modeled using linearized transfer function. For systems with strong nonlinear and frequent turbulence or wide range of operating point, the predictive control adopting linear model near operating point can no longer satisfy the requirements of control quality. Therefore, the nonlinear modeled predictive control has aroused wide concern.

A predictive control scheme for hydroturbine governor based on NNs is introduced in this paper. The control architecture consists of two NNs: an adaptive neuroidentifier (ANI) to track the plant and predict its output one-step-ahead and an adaptive neurocontroller (ANC) to produce the control signal. A scalar error is used in each sampling period to update the identifier and controller weights continuously in real-time. With a similar architecture, called indirect adaptive control [12], the use of a reference model is suggested. That is avoided in this work owing to the difficulties in choosing a proper reference model for the complex hydroturbine generator regulating system.

The effect of different amplitude step disturbances and trapezoidal shape reference signal (turbine power) are investigated in this paper. Also, a number of studies are performed to compare the performance of the proposed controller with that of the conventional PID controller under different operating conditions.

\section{Basic Plant Equations}

A simple layout of a hydropower plant shown in Figure 1 includes a reservoir, a penstock (high pressure), gate, a hydroturbine, and a generator. The function of the reservoir is to store water and thus develop a head. To start the turbine generator unit, water released from the reservoir flows through the penstock and reaches the turbine inlet. From here it enters into the scroll casing of the turbine that distributes the water evenly on the runner blades. The electromechanical power conversion takes place between the turbine runner and the generator mounted on the common shaft. The water flow to the turbine is regulated by the operation of wicket gates that in turn are controlled by the governor. The opening and closing of the gates are a function of the variable electrical load connected to the generator and the shaft speed.

The hydroturbine governing system consisting of the controller and the controlled plant is a complex, nonlinear, time varying, and non-minimum-phase system with fractional distributed parameters and uncertainties. The controlled plant includes a turbine, a penstock, a generator, and a load (Figure 2). In practice, a linearized (or linear) model is used to describe the plant for designing the control system. The dynamic characteristics of the plant are shown in Figure 2 [18].

2.1. Turbine. The characteristic equations of the Francis turbine are [19-21]

$$
\begin{gathered}
M_{t}=M_{t}(y, h, x), \\
Q=Q(y, h, x),
\end{gathered}
$$

where $M_{t}$ is torque of the turbine, $Q$ is flow rate, $y$ is gate opening, $h$ is head, and $x$ is speed. Taking Taylor series expansion of (1) and omitting the parts containing second- or higher-order derivatives, the linearized model can be expressed as follows in the neighborhood of certain operation:

$$
\begin{aligned}
& m_{t}=e_{y} y+e_{x} x+e_{h} h, \\
& q=e_{q y} y+e_{q x} x+e_{q h} h .
\end{aligned}
$$

The six transmission coefficients (as shown below) change as the gate opening changes:

$$
\begin{array}{ccc}
e_{y}=\frac{\partial m_{t}}{\partial y}, & e_{x}=\frac{\partial m_{t}}{\partial x}, & e_{h}=\frac{\partial m_{t}}{\partial h} \\
e_{q y}=\frac{\partial q}{\partial y}, & e_{q x}=\frac{\partial q}{\partial x}, & e_{q h}=\frac{\partial q}{\partial h}
\end{array}
$$

2.2. Penstock. The general expression of penstock, taking rigid water hammer, is described as

$$
h=-T_{w} \frac{d q}{d t}
$$

where $T_{w}$ is the water inertia time constant. 


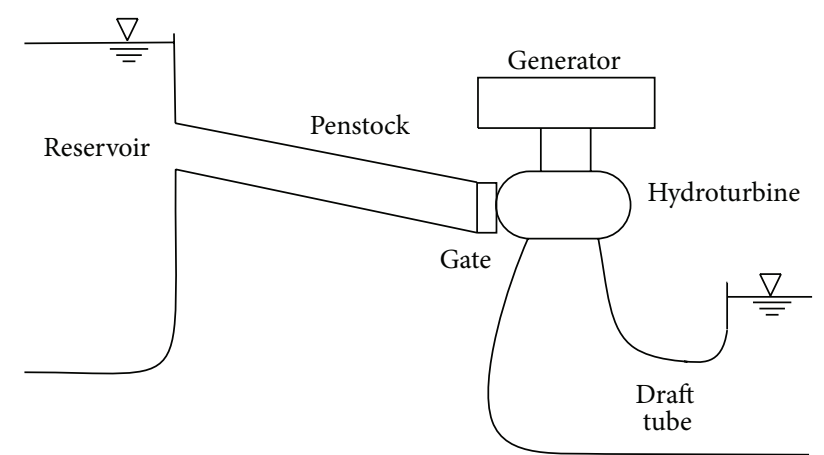

Figure 1: General layout of hydropower plant.

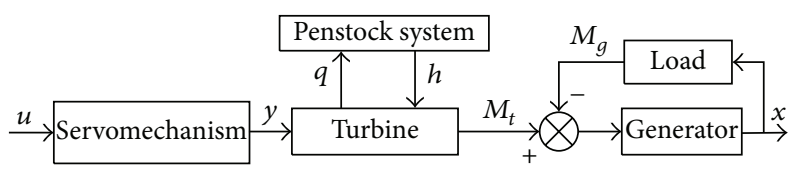

FIgURE 2: Hydroturbine generator regulating system.

2.3. Generator and Load. The characteristic equation of the generator and the load can be written as

$$
T_{a} \frac{d x}{d t}=m_{t}-m_{g}-e_{g} x,
$$

where $T_{a}$ is the unit inertia time constant, $m_{g}$ is the load torque, and $e_{g}$ is the load self-regulation factor, regarding the fluctuation caused by the change of the system frequency.

2.4. Servomechanism. Neglecting small time constants, the servomechanism can be expressed with a first-order equation:

$$
T_{y} \frac{d y}{d t}+y=u
$$

where $T_{y}$ is the servomotor response time and $u$ is the control signal.

\section{Controller Structure}

The structure of the controller is shown in Figure 3. It consists of two subnetworks. The first subnetwork is an adaptive onestep-ahead neuroidentifier that tracks the dynamic behaviour of the plant and identifies the plant in terms of its internal weights and the second one is an adaptive neurocontroller to provide the necessary control action in order to minimize certain cost function.

3.1. Adaptive Neuroidentifier. A multilayer perceptron neural network (MLPNN) structure is developed to model the nonlinear dynamic relationship between the gate position and the turbine mechanical power. Considering it is difficult to measure the turbine mechanical power, generator output power is measured instead to obtain the turbine mechanical power by

$$
P_{e}=\frac{P_{g}}{\eta}
$$

where $P_{e}$ is turbine mechanical power, $P_{g}$ is generator output power, and $\eta$ is generator efficiency.

The network transforms $n$ inputs to $m$ outputs through a nonlinear function $f: R^{n} \rightarrow R^{m}$. It is shown in [15] that an MLPNN with single hidden layer activated with sigmoid or hyperbolic tangent function can approximate any continuous function. Considering the nature of the dependence of the plant output on a finite number of past inputs, $u(t)$, and outputs, $y(t)$, the nonlinear relationship between the gate position and the turbine power can be represented in the form of predictor/identifier as

$$
\begin{aligned}
& y_{(k+1)} \\
& \quad=f\left[y_{(k)}, y_{(k-1)}, \ldots, y_{(k-n+1)}, u_{(k)}, u_{(k-1)}, \ldots, u_{(k-m+1)}\right] .
\end{aligned}
$$

In this case a popular MLPNN with single hidden layer activated with sigmoid function and back propagation learning has been used to develop the predictor of nonlinear relationship model (8). The input vector to the ANI is

$$
\begin{aligned}
& {\left[p_{(k)}, p_{(k-1)}, \ldots, p_{(k-n+1)},\right.} \\
& \left.u_{(k)}, u_{(k-1)}, \ldots, u_{(k-m+1)}\right],
\end{aligned}
$$

where $p(k)$ is the turbine mechanical power and $u(k)$ is the gate position at the time step $k$. The output is the predicted turbine mechanical power $\widehat{p}_{(k+1)}$, at time step $(k+1)$. For a finite number of past inputs, $u(t)$, and outputs, $p(t)$, the nonlinear relationship between the control output (gate position) and turbine power can be represented in the form of an identifier as shown in Figure 4.

The input vector to the ANI is scaled in the range of $[-1,+1]$ before being applied to the network. The cost function used for the ANI is

$$
J_{i}(k)=\frac{1}{2} e_{i}(k)^{2}=\frac{1}{2}[p(k)-p \widehat{(k)}]^{2} .
$$

The weights are updated as

$$
w_{i}(k)=w_{i}(k-1)-\eta_{i} \frac{\partial J_{i}(k)}{\partial w_{i}(k)},
$$

where $w_{i}$ is the matrix of identifier weights at time step $k$ and $\eta_{i}$ is the learning rate for the ANI. This cost function is minimized by back propagating the scalar error. In each sampling period, the input and the output of the plant are sampled and the input vector to the identifier is formed as in (9). Then the error between the actual and predicted outputs of the plant, a scalar value, is back propagated through the identifier to update the weights of the network $\left(w_{i}(k)\right)$. This process is repeated every sampling period that in turn results in an adaptive approach to predict one-step-ahead of the output of the plant. The use of just one error value for back propagation simplifies the training algorithm by reducing computation time. 


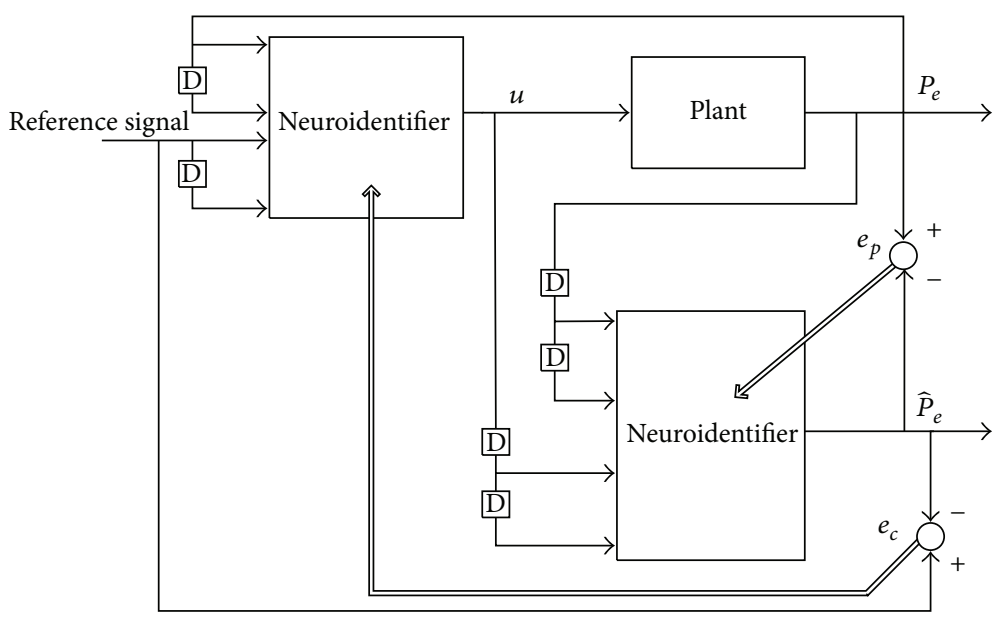

FIGURE 3: Schematic of control structure.

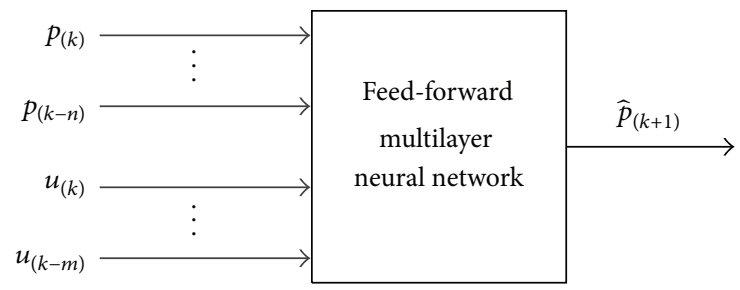

FIgURE 4: Neuroidentifier.

3.2. Adaptive Neurocontroller. Taking advantage of the recognized universal approximation properties of NN, a nonlinear plant in the MLPNN form is obtained as discussed before. Based on the neural model, a predictive control strategy is implemented by an adaptive neurocontroller. The input vector to the ANC is

$$
\begin{gathered}
{\left[p_{(k)}, p_{(k-1)}, \ldots, p_{(k-p+1)},\right.} \\
\left.r_{(k)}, r_{(k-1)}, \ldots, r_{(k-q+1)}\right],
\end{gathered}
$$

where $r(k)$ is the reference input signal at time step $k$. The output of the ANC is the control action $u(k)$ at time step $k$. The inputs to the ANC are also scaled in the range of $[-l,+l]$. The cost function for the ANC is considered as

$$
\begin{aligned}
J_{c}(k) & =\frac{1}{2}\left[e_{c}(k)^{2}+h u(k)\right]^{2} \\
& =\frac{1}{2}[r(k)-p \widehat{(k)}]^{2}+\frac{h}{2} u(k)^{2},
\end{aligned}
$$

where $r(k)$ is the reference input signal at time step $k$ and $h$ is a tuning parameter that is used to improve the plant output dynamic characteristics. By taking $h$ larger than zero, a penalty factor is applied to the control operation that helps the tuning of the dynamic trajectory and optimizing the overshoot and the settling time of the response curve. The weights of the controller $w_{c}(k)$ are updated as

$$
w_{c}(k)=w_{c}(k-1)-\eta_{c} \frac{\partial J_{c}(k)}{\partial w_{c}(k)} .
$$

Using (13) and (14) $J_{c}(k)$ is minimized in each sampling period. As depicted in (9) and (12), the states of the plant are not required for the implementation of the ANI and ANC, and only input-output data are needed. This greatly simplifies the implementation of the control process.

\section{Training Process}

The success implementation of the control algorithm presented in Section 3 highly depends on the accuracy of the identifier in tracking dynamic plant. For this reason, the ANI is initially trained off-line before being used in the final configuration. The training is performed over a wide range of operating conditions for the generating unit. After the off-line training stage, the ANI is employed in the system. Further updating of the weights of ANI and ANC is performed in each sampling period by employing the on-line version of the back propagation method. This enables the controller to track the plant variations as they occur to yield the optimum performance. The main steps of the adaptive predictive control algorithm are listed as follows, and the algorithm flowchart is shown in Figure 5.

(1) At time step $k, p(k)$ is sampled.

(2) Compute $\widehat{p}_{(k)}$ using ANI and its input vector (9).

(3) Calculate the error between $p(k)$ and $\widehat{p}_{(k)}$; then update the weights of the ANI to minimize $J_{i}(k)$ utilizing the back propagation method and the gradient descent algorithm.

(4) The output of the controller $u(k)$ is computed.

(5) Using input vector (9), the predicted $\widehat{p}_{(k+1)}$ is computed by the ANI with weights updated in step (3).

(6) Based on $\widehat{p}_{(k+1)}$ and reference signal, weights of the ANC are updated, minimizing $J_{c}(k)$ by utilizing the back propagation method and the gradient descent algorithm.

In step (3) above, the training is straightforward since the error at the output of the ANI is obtained. However, in 


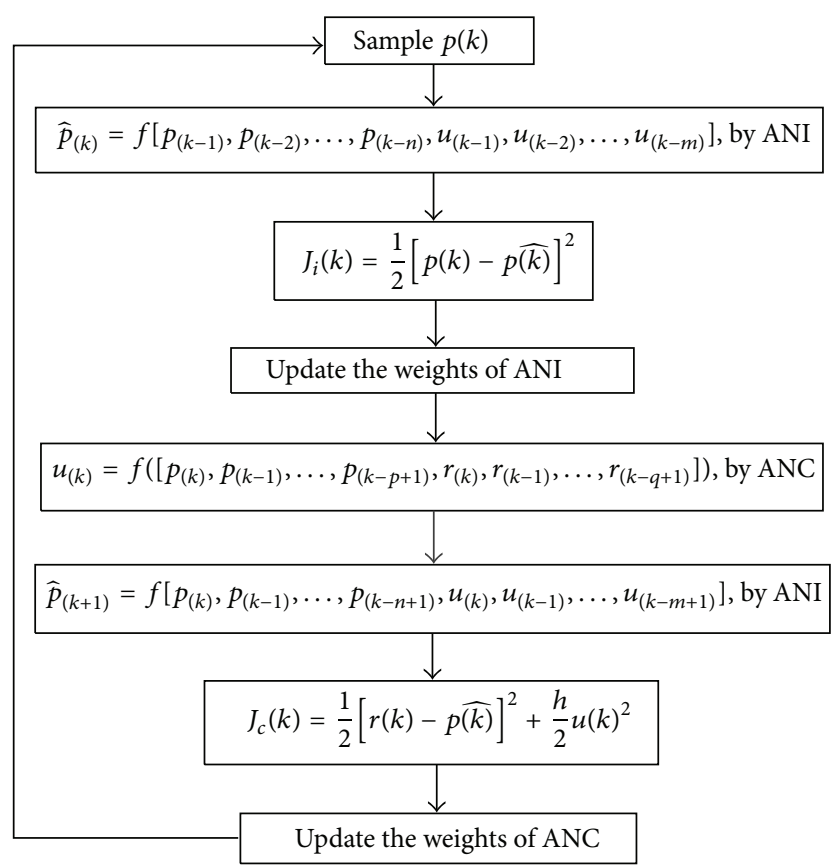

FIGURE 5: The flowchart of the adaptive predictive control algorithm.
TABLE 1: Transmission coefficients as a function of gate opening.

\begin{tabular}{lcccccc}
\hline $\begin{array}{l}\text { Operating } \\
\text { point } \\
\text { number }\end{array}$ & $\begin{array}{c}\text { Gate } \\
\text { opening \% }\end{array}$ & $e_{y}$ & $e_{h}$ & $e_{x}$ & $e_{q y}$ & $e_{q h}$ \\
\hline 1 & 43.74 & 2.867 & 0.526 & -0.353 & 1.674 & 0.232 \\
2 & 47.52 & 2.562 & 0.679 & -0.455 & 1.541 & 0.262 \\
3 & 51.26 & 2.278 & 0.814 & -0.545 & 1.416 & 0.290 \\
4 & 55 & 2.016 & 0.934 & -0.626 & 1.300 & 0.315 \\
5 & 58.74 & 1.766 & 1.039 & -0.696 & 1.191 & 0.338 \\
6 & 62.52 & 1.654 & 1.132 & -0.759 & 1.090 & 0.360 \\
7 & 66.26 & 1.333 & 1.212 & -0.813 & 0.997 & 0.380 \\
8 & 70 & 1.146 & 1.281 & -0.859 & 0.913 & 0.397 \\
9 & 73.74 & 0.974 & 1.340 & -0.898 & 0.837 & 0.414 \\
10 & 77.52 & 0.822 & 1.391 & -0.932 & 0.768 & 0.429 \\
11 & 81.26 & 0.691 & 1.433 & -0.961 & 0.708 & 0.443 \\
12 & 85 & 0.578 & 1.468 & -0.984 & 0.655 & 0.455 \\
13 & 88.74 & 0.484 & 1.498 & -1.000 & 0.611 & 0.467 \\
14 & 92.52 & 0.409 & 1.523 & -1.020 & 0.574 & 0.478 \\
15 & 96.26 & 0.353 & 1.544 & -1.030 & 0.546 & 0.489 \\
16 & 100 & 0.317 & 1.563 & -1.047 & 0.526 & 0.499 \\
\hline
\end{tabular}

step (6) the training is difficult since the error at the output of the ANC is not provided. In this case, first, the weights of the ANI are frozen and the error between the desired and predicted plant output is back propagated through the ANI. Then, the back propagated signal at the input of the ANI is further propagated through the ANC, making necessary changes to the controller weights. In other words, for adapting weights of the controller, the identifier acts as a channel to convey the error from the output of the identifier to the output of the controller. This evaluates the need to have the identifier. The error is used to train the ANI and the ANC.

\section{Simulation Results}

The plant model is simulated using the mathematical model given in Section 2. The values of plant parameters used are

$$
\begin{aligned}
& T_{a}=9.06 \mathrm{~s}, \quad T_{w}=1.27 \mathrm{~s}, \quad T_{r}=0.15 \mathrm{~s}, \\
& \xi=0.2, \quad T_{1}=0.02 \mathrm{~s}, \quad T=0.04 \mathrm{~s}, \\
& s_{1}=0.00167, \quad i_{x}=0.04 \%,
\end{aligned}
$$

where $T_{r}$ is penstock reflection time, $\xi$ is damping constantinfinite bus tie, $T_{1}$ is servomotor response time, $T$ is the sampling period, $s_{1}$ is speed limit on control in a governor, and $i_{x}$ is dead band. The transmission coefficients (except $e_{q x}$ which is usually equal to 0 ) have different values for the different gate openings. A set of transmission coefficients corresponding to the gate opening for a turbine located in Ouyanghai hydropower plant in China is given in Table 1. The design water head of Ouyanghai hydropower station is 37.6 meters, and design flow is $38.4 \mathrm{~m}^{3} / \mathrm{s}$. Turbine model is chosen as HL123-LJ-225, and rated capacity and voltage of the turbine are $1200 \mathrm{kw}$ and $10.5 \mathrm{kv}$, respectively.
The Simulation Toolbox SIMULINK of MATLAB is utilized to develop plant model and generate data. The absolute value of pseudorandom binary signal is applied to the input to represent the variation of gate position, and the corresponding turbine mechanical power is obtained. The data collected (input and output) are divided into two sets: one set is for training the NN and the other set is for validation.

An input-output identifier model and control strategy is established and its parameters are set as follows. The number of time delays used for the input of ANI and ANC is set to 3; that is, $m, n, p$, and $q$ in (8), (9), and (12) are all set to 3. This means that both the ANI and the ANC have 6 inputs. There is one hidden layer of 8 neurons with sigmoid nonlinearity and an output layer with one linear neuron, for both the ANI and the ANC. Initial weights of the ANC lie in $[-0.1,+0.1]$, chosen randomly at the beginning of the process. The initial weights of the ANI are set to those obtained from off-line training stage of the ANI as discussed before. The learning rate for the ANI and the ANC is 0.01 and 0.03 , respectively. The value of the penalty factor $h$ is set between 0.1 and 2.0.

The quadratic programming problem in (13) is solved by using the quadprog function in the Optimization Toolbox of MATLAB. The MLP network algorithm is realized by using the functions of Train, Init, and Sim in the Neural Network Toolbox of MATLAB.

As the control parameters have been determined as discussed before, the performance of the proposed adaptive neural predictive control is simulated on a large amplitude step and trapezoidal wave-shape reference signals. These reference signals may represent the nature of load changes. The turbine gate opening and power output are shown in Figures 6-9 on different reference signals and various values of penalty factor $h$. 


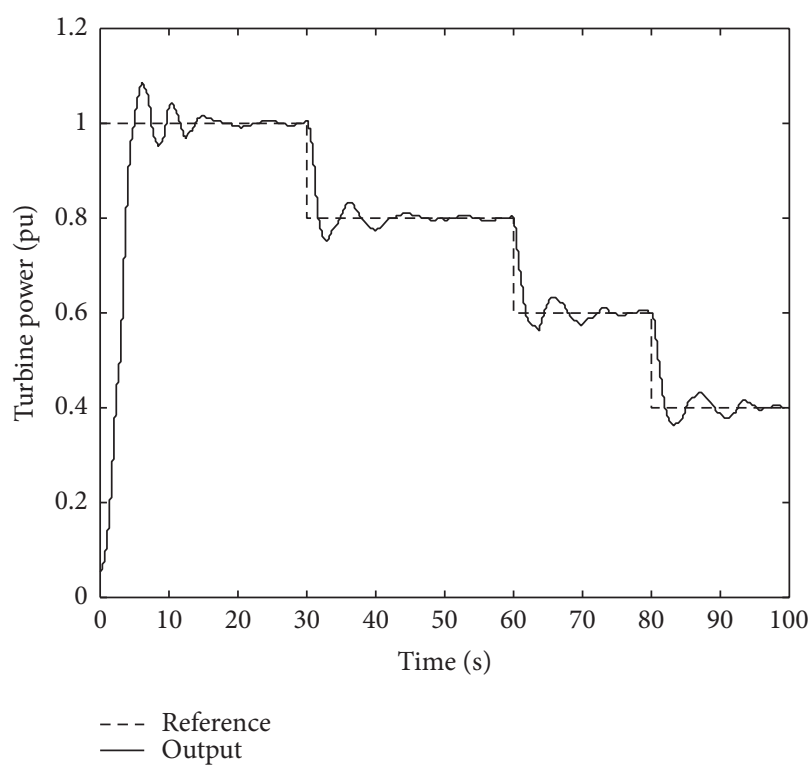

(a) Turbine power output response

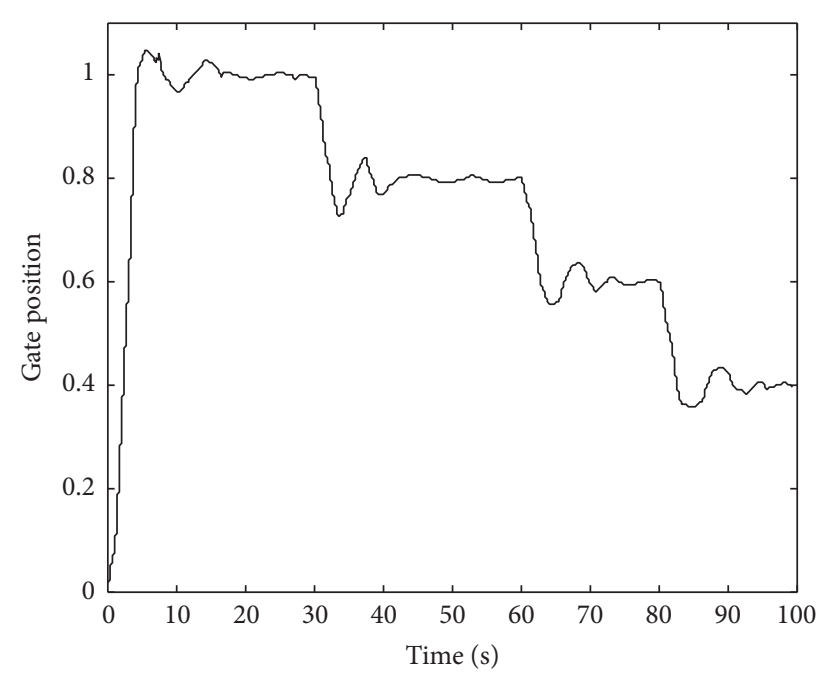

(b) Turbine gate opening response

FIGURE 6: Response to large amplitude step in turbine power reference, $h=2.0$.

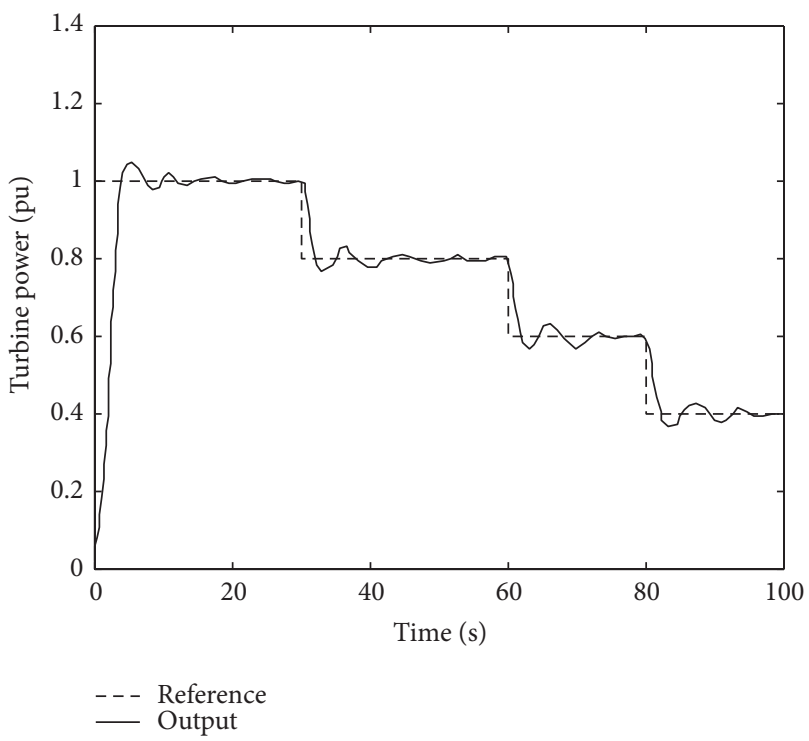

(a) Turbine power output response

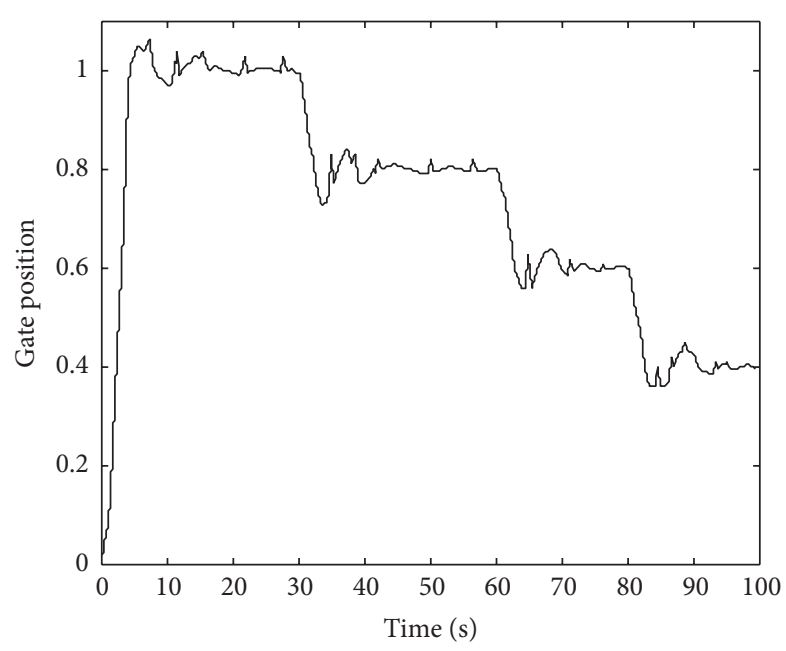

(b) Turbine gate opening response

FIgURE 7: Response to large amplitude step in turbine power reference, $h=0.18$.

The response shows a non-minimum-phase characteristic. The turbine power response on the large amplitude step signal follows more or less closely. It is evident that despite a large change in the operating conditions the controller still provides good results because of the adaptation process. However, the gate position is observed to exhibit large fluctuation on step change with $h=0.18$. This would cause undue actuator wear. The optimization seems to be insensitive to variations in control penalty factor. In the case of trapezoidal wave signal, the controlled variable reaches its steady-state value extremely fast with a little offset (i.e., output power overlaps the reference signal) and gate position variation within limits is demonstrated.

A number of studies have been performed to compare the quality of the proposed adaptive predictive controller with those of the conventional PID controller.

Generally, the conventional governors adopt a PI or PID control law. Figure 10 is the illustration of the conventional 

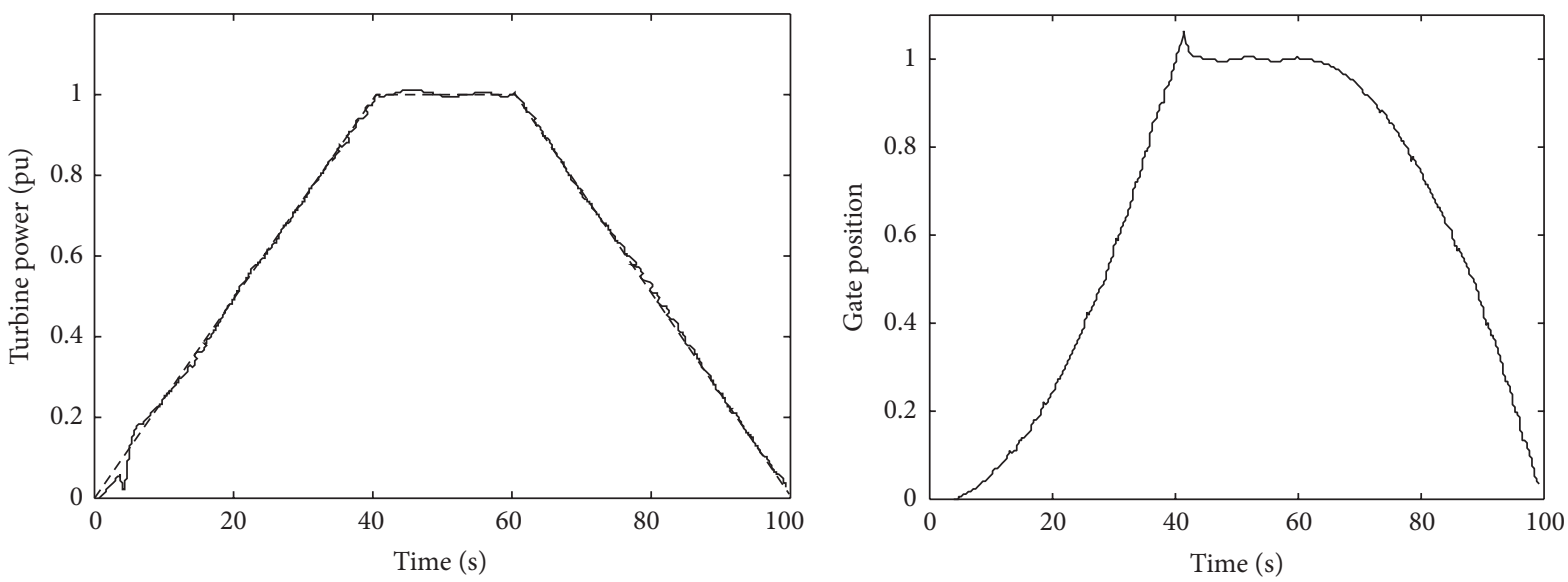

\section{- - - Reference}

Output

(a) Turbine power output response

(b) Turbine gate opening response

FIgURE 8: Response to trapezoidal wave reference, $h=2.0$.

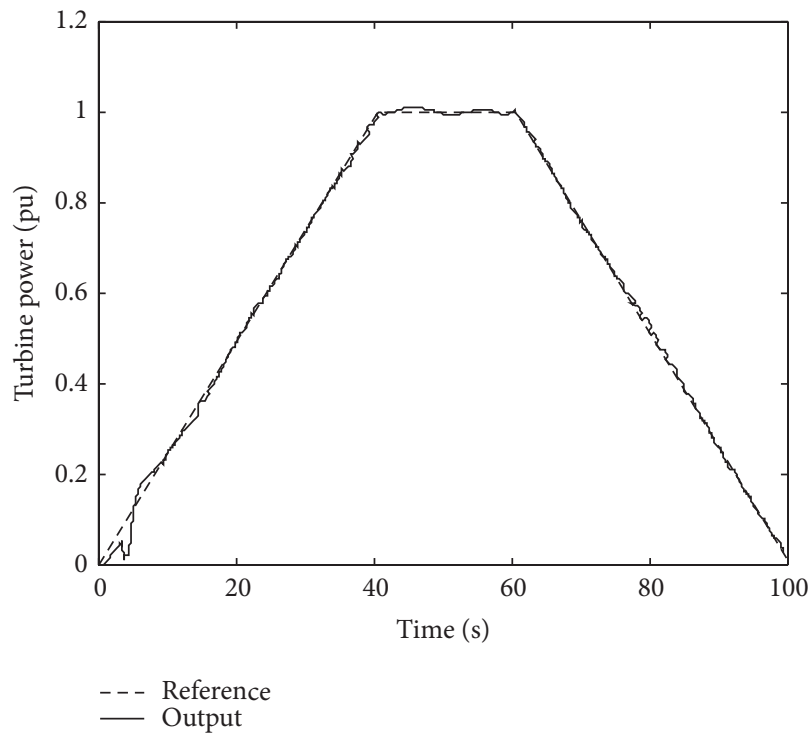

(a) Turbine power output response

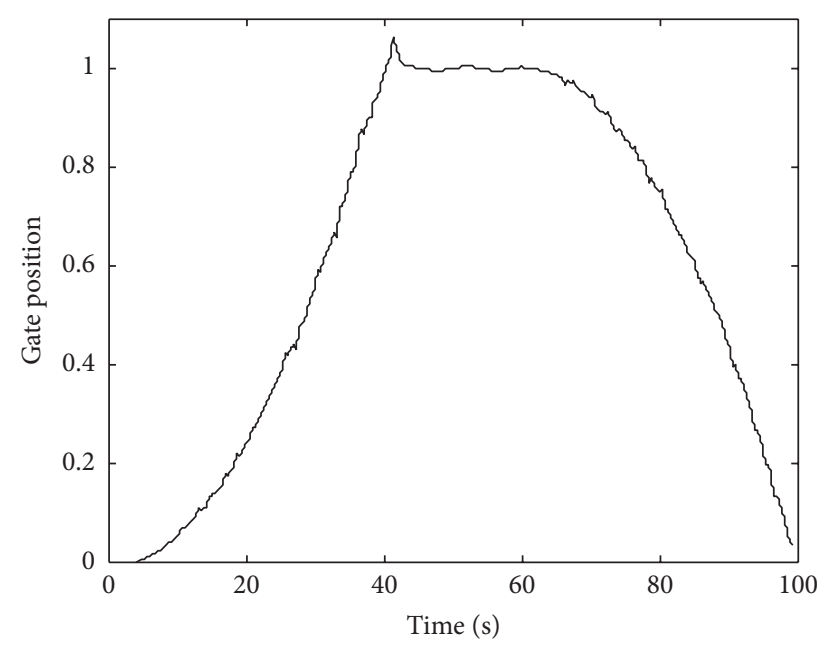

(b) Turbine gate opening response

FIGURE 9: Response to trapezoidal wave reference, $h=0.18$.

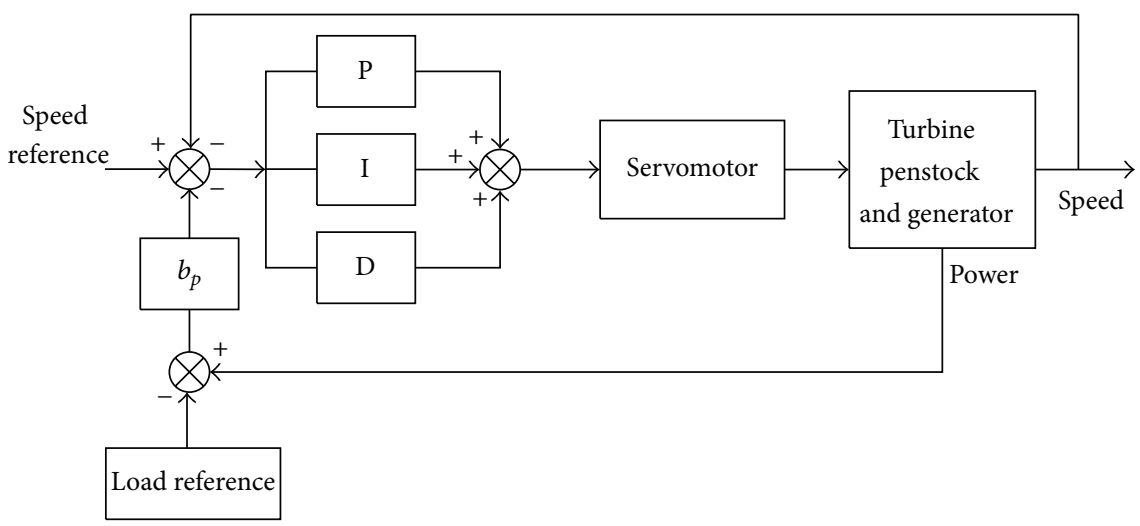

FIgURE 10: Illustration of the conventional load-change method. 
load changing process, where bp is permanent speed droop. It plays an important role in adjusting the load of the unit and the load distribution among all units in the power system.

Regarding the adjustments of PID parameters, the following formulas are often used by experienced engineers.

For PI controller,

$$
T_{n}=0, \quad b_{t}=\frac{2.6 T_{w}}{T_{a}}, \quad T_{d}=6 T_{w} ;
$$

for PID controller,

$$
T_{n}=0.5 T_{w}, \quad b_{t}=\frac{1.5 T_{w}}{T_{a}}, \quad T_{d}=3 T_{w}
$$

where $T_{n}$ is the derivative time constant, $b_{t}$ is the transient speed droop, and $T_{d}$ is the damping time constant. Their relation with proportional, integral, and differential gain coefficients of a continuous PID controller is

$$
k_{p}=\frac{T_{d}+T_{n}}{b_{t} T_{d}}, \quad k_{i}=\frac{1}{b_{t} T_{d}}, \quad k_{d}=\frac{T_{n}}{b_{t}} .
$$

According to these empirical formulae, the parameters of the conventional PID controller are chosen as below:

$$
k_{p}=5.56, \quad k_{i}=1.25, \quad k_{d}=3.03, \quad b_{p}=0 .
$$

The proposed adaptive neuropredictive controller adopts the parameters determined as discussed above and the value of the penalty factor $h$ is set to 2.0 .

Step increases of $10 \%$ and $80 \%$ in power reference are introduced and the system responses with the conventional PID controller (CPID) and the proposed adaptive neuropredictive controller (PAPC) are shown in Figures 11-14. The dashed curves represent the response of CPID, and the solid lines represent the response of PAPC.

It is observed from Figure 11 that, with the conventional PID controller, the settling time, defined as the moment when the system error is less than $5 \%$ of the input reference signal, is $8.9 \mathrm{~s}$ and the overshoot is $18.3 \%$, but, with the adaptive predictive controller, the setting time is $7.2 \mathrm{~s}$ and overshoot is $13.1 \%$. It can be seen from Figure 12 that the settling time with the conventional PID controller is $28.3 \mathrm{~s}$ and the overshoot is $37.4 \%$, but, with the adaptive predictive controller, the setting time is $7.4 \mathrm{~s}$ and overshoot is $13.5 \%$.

From the responses for $10 \%$ and $80 \%$ step changes in power reference, it can be seen that the conventional PID controller only provides acceptable performance for a small disturbance rather than the large disturbance. Even for a small disturbance, the proposed adaptive predictive controller is better than the conventional one regarding the speed and the overshoot of response of the process.

This is logically correct because of the existence of nonlinearities in a hydroturbine governing system. These nonlinearities can be divided into two parts. The first comes from the turbine's nonlinear characteristics that depend on the operating point. It can be clearly seen from (2) of the turbine that the six transmission coefficients change

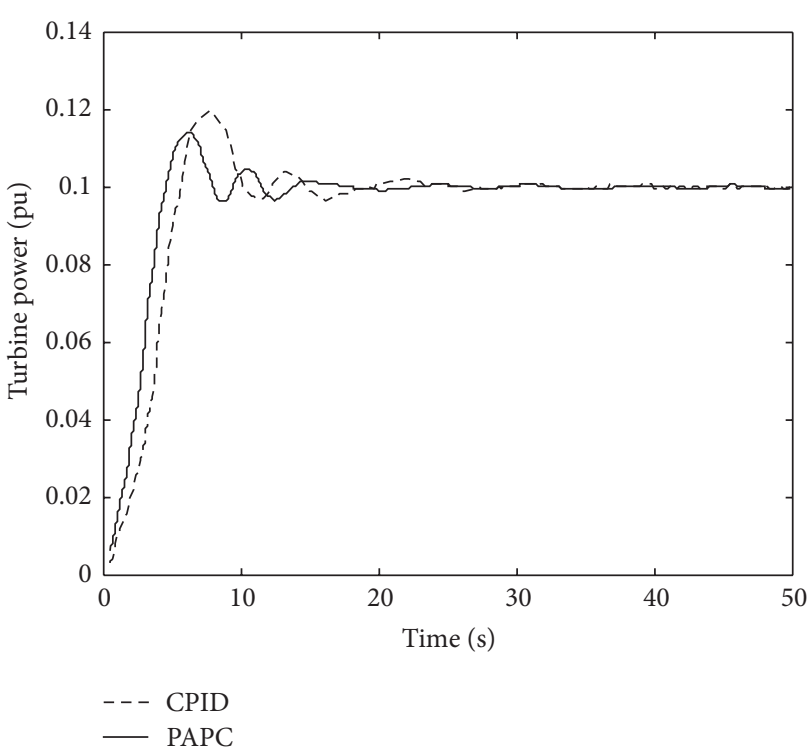

FIGURE 11: Turbine power output response to a 10\% step in reference.

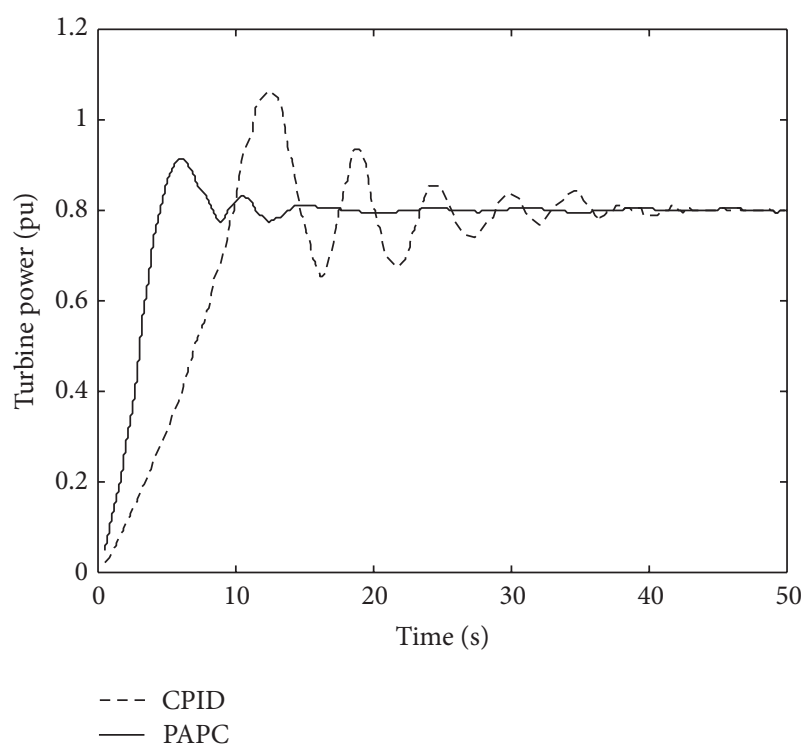

FIGURE 12: Turbine power output response to an $80 \%$ step in reference.

as the gate opening changes. The second part is due to the nonlinear factors such as magnitude limit on control, dead band, and speed limit on control. Therefore, in the conventional PID controller, the regulation parameters that give good performance for small disturbance are no longer optimal for the large disturbance. In the case of a small disturbance, the effect of nonlinearity is negligible and the conventional controller is designed based on the linear control theory, but, in the case of a large disturbance, the effect of nonlinearity cannot be neglected. The plant has changed; however the control parameters of the conventional PID controller have not changed. Therefore, it is unable to maintain adequate performance levels. In contrast, it can be 


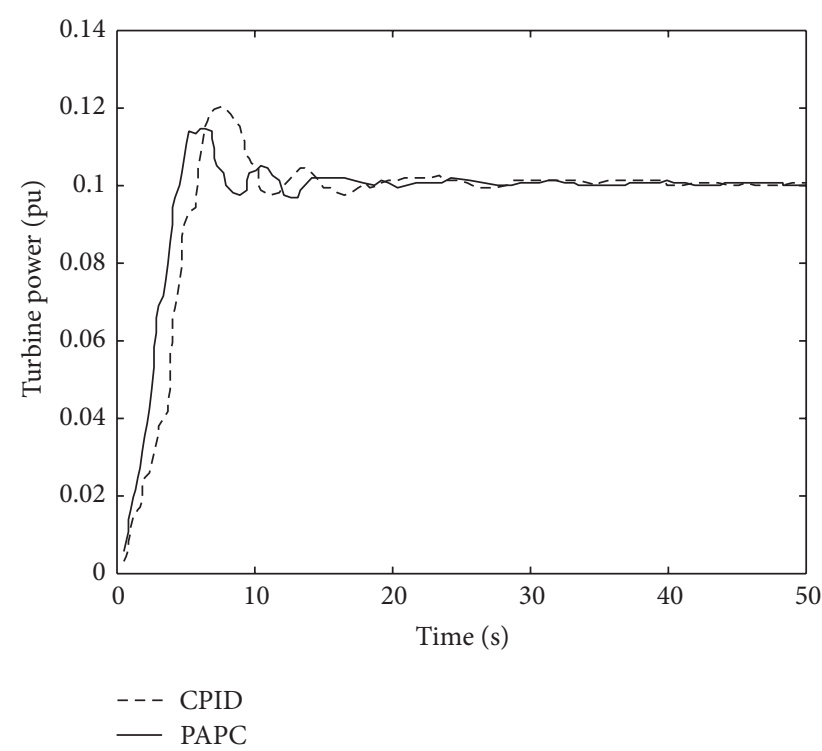

FIGURE 13: Gate opening response to a 10\% step in reference.

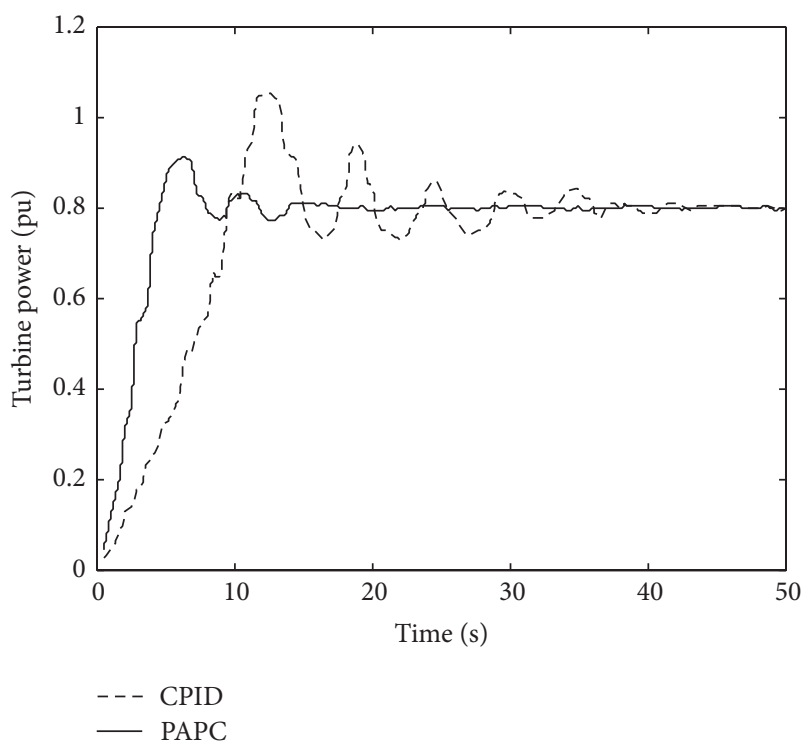

FIGURE 14: Gate opening response to an $80 \%$ step in reference.

seen that the proposed adaptive predictive controller has a good response for both small and large disturbances, because it considers the nonlinear nature of the plant and has the ability to adjust its own parameters on-line according to the working environment. Even for the small disturbance, the proposed adaptive predictive controller considers the plant nonlinearity as well, while it is usually ignored in the conventional PID controller. Therefore, the proposed adaptive predictive controller has better control performance than the conventional one in regard to the speed and the overshoot of the process response.

\section{Conclusions}

An adaptive neuropredictive control for hydroturbine governor is presented in this paper. The back propagation network with on-line learning is used in the proposed method. The controller introduced in this work inherits the general advantages of neural networks such as high speed, generalization capability, and fault tolerance as well as adaptation (learning) property. This method features the simple structure and the nonrequirement for a large number of neurons in the implementation. The learning algorithm is simplified by employing a single element error vector. The controller weights are updated directly in an on-line mode from the inputs and the outputs of the generator, and the states of the system are not necessarily determined. Simulation results for the large amplitude step and trapezoidal waveshape reference signals show that the proposed adaptive predictive controller can adaptively improve the dynamic performance of the system. By comparing the performance of the proposed adaptive predictive controller with that of the conventional PID controller, it is found that the proposed adaptive predictive controller is not only simple but also robust, and it features strong adaptivity as well.

\section{Conflict of Interests}

The authors declare that there is no conflict of interests regarding the publication of this paper.

\section{Acknowledgment}

The authors acknowledge the financial supports from the National Natural Science Foundation of China under Grant no. 51379160 .

\section{References}

[1] N. Kishor, R. P. Saini, and S. P. Singh, "A review on hydropower plant models and control," Renewable and Sustainable Energy Reviews, vol. 11, no. 5, pp. 776-796, 2007.

[2] H. Fang, L. Chen, and Z. Shen, "Application of an improved PSO algorithm to optimal tuning of PID gains for water turbine governor," Energy Conversion and Management, vol. 52, no. 4, pp. 1763-1770, 2011.

[3] D. G. Ramey and J. W. Skooglund, "Detailed hydro governor representation for system stability studies," IEEE Transactions on Power Apparatus and Systems, vol. 89, no. 1, pp. 106-112, 1970.

[4] Q. Chen and Z. Xiao, "Dynamic modeling of hydroturbine generating set," in Proceedings of the IEEE International Conference on Systems, Man and Cybernetics, pp. 3427-3430, October 2000.

[5] C. Jiang, Y. Ma, and C. Wang, "PID controller parameters optimization of hydro-turbine governing systems using deterministic-chaotic-mutation evolutionary programming (DCMEP)," Energy Conversion and Management, vol. 47, no. 9-10, pp. 12221230, 2006.

[6] D. Zhang and L. Yu, "Exponential state estimation for Markovian jumping neural networks with time-varying discrete and distributed delays," Neural Networks, vol. 35, pp. 103-111, 2012. 
[7] P. Hušek, "PID controller design for hydraulic turbine based on sensitivity margin specifications," International Journal of Electrical Power and Energy Systems, vol. 55, pp. 460-466, 2014.

[8] Z. Li and O. P. Malik, "An orthogonal test approach based control parameter optimization and its application to a hydroturbine governor," IEEE Transactions on Energy Conversion, vol. 12, no. 4, pp. 388-393, 1997.

[9] A. Soós and O. P. Malik, "An $\mathrm{H}_{2}$ optimal adaptive power system stabilizer," IEEE Transactions on Energy Conversion, vol. 17, no. 1, pp. 143-149, 2002.

[10] H. Fang, L. Chen, N. Dlakavu, and Z. Shen, "Basic modeling and simulation tool for analysis of hydraulic transients in hydroelectric power plants," IEEE Transactions on Energy Conversion, vol. 23, no. 3, pp. 834-841, 2008.

[11] M. Mahmoud, K. Dutton, and M. Denman, "Dynamical modelling and simulation of a cascaded reserevoirs hydropower plant," Electric Power Systems Research, vol. 70, no. 2, pp. 129139, 2004.

[12] P. Shamsollahi and O. P. Malik, "An adaptive power system stabilizer using on-line trained neural networks," IEEE Transactions on Energy Conversion, vol. 12, no. 4, pp. 382-387, 1997.

[13] I. Eker, "Governors for hydro-turbine speed control in power generation: a SIMO robust design approach," Energy Conversion and Management, vol. 45, no. 13-14, pp. 2207-2221, 2004.

[14] Y.-C. Cheng, L.-Q. Ye, F. Chuang, and W.-Y. Cai, "Anthropomorphic intelligent PID control and its application in the hydro turbine governor," in Proceedings of the International Conference on Machine Learning and Cybernetics, pp. 391-395, November 2002.

[15] G. Cybenko, "Approximation by superpositions of a sigmoidal function," Mathematics of Control, Signals, and Systems, vol. 2, no. 4, pp. 303-314, 1989.

[16] Z. Shen, Governing of Hydroturbine, China Water Publishing Press, Beijing, China, 3rd edition, 1998.

[17] D. Jones and S. Mansoor, "Predictive feedforward control for a hydroelectric plant," IEEE Transactions on Control Systems Technology, vol. 12, no. 6, pp. 956-965, 2004.

[18] K.-I. Funahashi, "On the approximate realization of continuous mappings by neural networks," Neural Networks, vol. 2, no. 3, pp. 183-192, 1989.

[19] C. D. Vournas and A. Zaharakis, "Hydro turbine transfer functions with hydraulic coupling," IEEE Transactions on Energy Conversion, vol. 8, no. 3, pp. 527-532, 1993.

[20] Z. Xiao, S. Wang, H. Zeng, and X. Yuan, "Identifying of hydraulic turbine generating unit model based on neural network," in Proceedings of the 6th International Conference on Intelligent Systems Design and Applications (ISDA '06), vol. 1, pp. 113-117, October 2006.

[21] J. Chang, B. Liu, and W. Cai, "Nonlinear simulation of hydroturbine governing system based on neural network," in Proceedings of the IEEE International Conference on Systems, Man and Cybernetics, pp. 784-787, October 1996. 


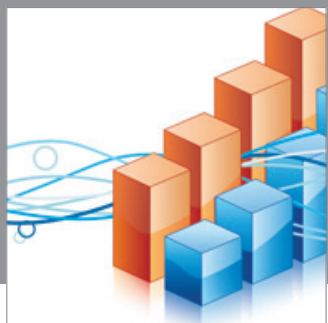

Advances in

Operations Research

mansans

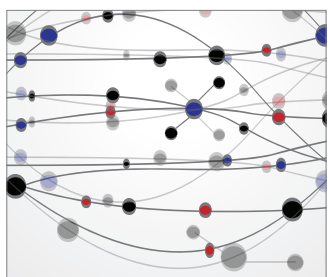

The Scientific World Journal
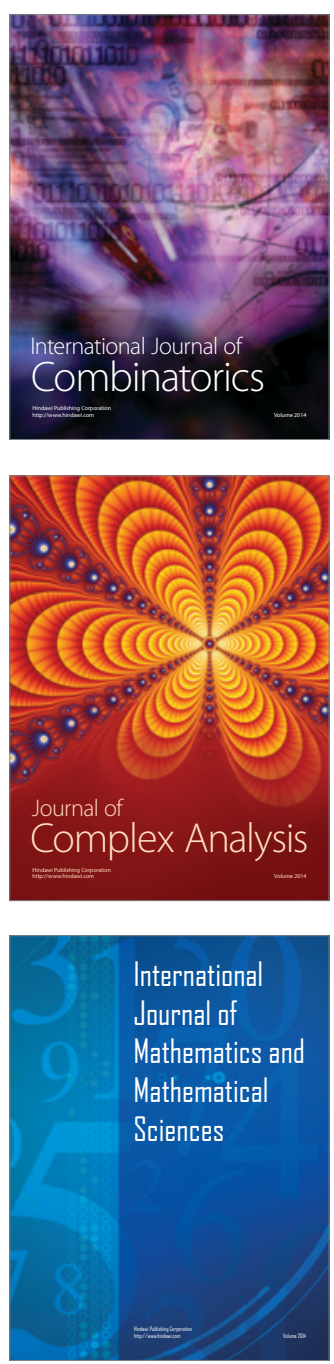
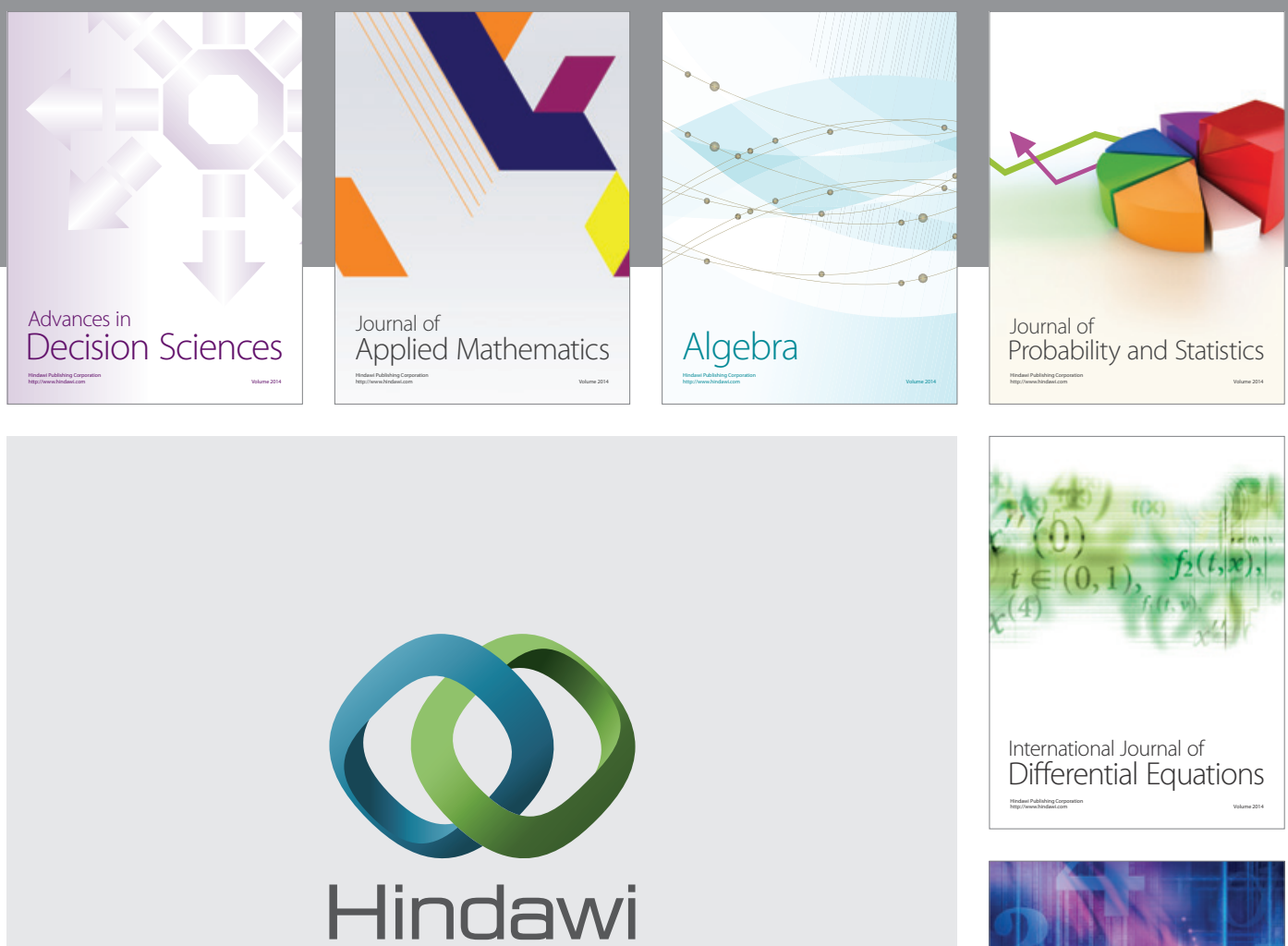

Submit your manuscripts at http://www.hindawi.com
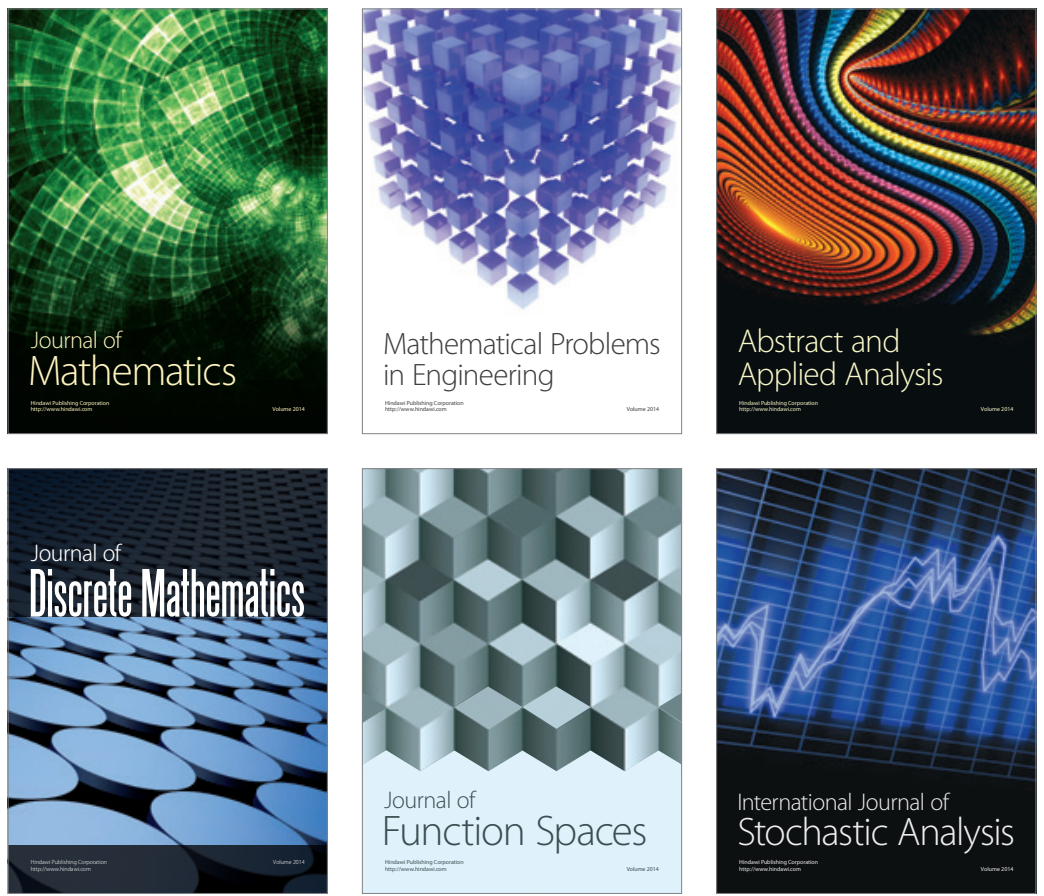

Journal of

Function Spaces

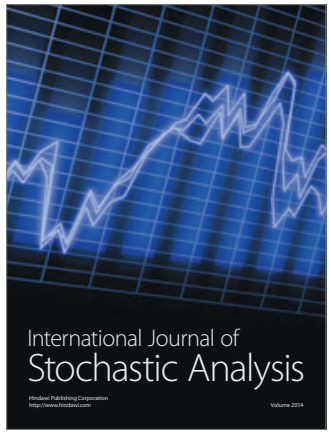

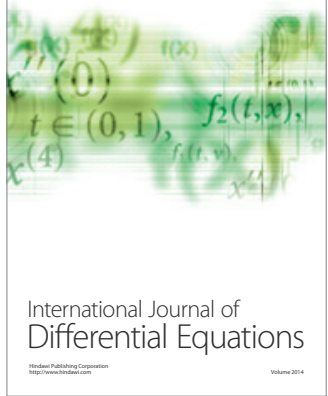
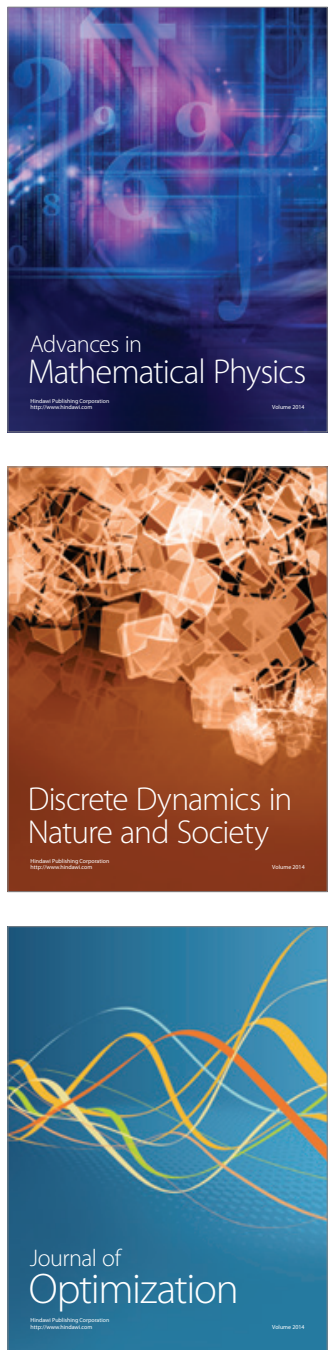